\title{
Mapeamento Sistemático sobre Metodologias e Ferramentas de apoio para o Ensino de Programação
}

\author{
Lucas Blatt', Valdecir Becker', Alexandre Magno e Silva Ferreira ${ }^{1,2}$ \\ ${ }^{1}$ Programa de Pós-Graduação em Computação, Comunicação e Artes (PPGCCA) \\ Centro de Informática, Universidade Federal da Paraíba (UFPB) \\ Caixa Postal 58051-900 - Cidade Universitária - João Pessoa, PB - Brasil \\ ${ }^{2}$ Departamento de Música - Universidade Federal da Paraíba (UFPB) \\ Caixa Postal 58051-900 - Cidade Universitária - João Pessoa, PB - Brasil \\ lucas.blatt@lavid.ufpb.br, valdecir@ci.ufpb.br, basstrombone@uky.edu
}

\begin{abstract}
A major challenge in undergraduate courses in Computer Science is programming teaching. Several methods and tools are proposed to facilitate and maximize the ability to students to learn. However, students face learning problems. This work presents a systematic mapping of the Brazilian literature about methodologies and tools used in programming teaching. The results show a great evolution of researches in the area of basic education, providing solid basis for undergraduate courses of Computer Science, with a number of researches pointing to a tendency in using gamified applications.
\end{abstract}

Resumo. Um grande desafio em cursos de graduação em Ciências da Computação é o ensino de programação. Vários métodos e ferramentas têm sido propostos para facilitar e maximizar a capacidade do aluno em aprender. No entanto, os alunos acabam enfrentando obstáculos que dificultam o processo de ensino aprendizagem. Este trabalho apresenta um mapeamento sistemático da literatura brasileira sobre as metodologias e ferramentas utilizadas no ensino de programação. Os resultados mostram uma grande evolução de pesquisas na área e a educação básica, oferecendo alicerce para cursos superiores de computação, com o maior número de pesquisas e uma tendência em usar aplicações gamificadas.

\section{Introdução}

Com o advento da tecnologia e aplicabilidade nas mais diversas áreas do conhecimento, o ensino da programação de computadores é um tema estudado e discutido devido às diversas formas de se abordar o aspecto da aprendizagem. A importância do ensino da programação para novos estudantes da área não está somente no conteúdo abordado, mas também na capacidade de transpor alguns conceitos conhecidos de outras áreas para uma linguagem de programação [37].

Diversas são as maneiras ou estratégias que podem ser adotadas no ensino de programação em cursos superiores nas mais diversas áreas. Um número cada vez maior de professores busca maneiras motivadoras para transmitir para os alunos os conceitos que envolvem as técnicas de programação. Segundo [15], a estratégia pedagógica mais comum de ensino de programação se 
constitui em aulas tradicionalmente teóricas, que são complementadas em laboratórios onde os alunos aplicam os conceitos estudados, através de exercícios utilizando uma linguagem de programação. Embora esta seja uma estratégia importante dentro do processo de ensino da programação, ela não deve ser isolada, pois podem acabar por gerar um processo de reprodução daquilo que foi transmitido em sala de aula, em detrimento a real construção do conhecimento.

As principais dificuldades encontradas durante o processo de aprendizagem são reflexos de diversos problemas, como lógica de programação ou a não familiaridade com um determinado paradigma [15]. É fundamental haver, durante o processo de aplicação dos conceitos, maior foco na solução do problema, levando em consideração a vivência e os conhecimentos trazidos previamente pelos estudantes.

Em se tratando das Diretrizes Curriculares de Cursos da Área de Computação e Informática [12], observamos que "o estudo de programação não se restringe ao estudo de linguagens de programação. As linguagens de programação constituem-se em uma ferramenta de concretização de software, que representa o resultado da aplicação de uma série de conhecimentos que transformam a especificação da solução de um problema em um programa de computador que efetivamente resolve aquele problema." Esta abordagem reforça o princípio de que o importante para o aluno é saber aplicar de maneira coerente as técnicas e abordagens estudadas através dos paradigmas de programação.

Segundo [51], de maneira objetiva, as diretrizes que regem o ensino da programação, em conjunto com as necessidades de formação profissional, torna-se claro que a interdisciplinaridade entre diferentes conteúdos que compõem o eixo do ensino de programação é uma forma de apresentar ao aluno como diversos conteúdos de disciplinas distintas podem ser integrados para a solução de um problema. Ou seja, dentro do aspecto abordado neste trabalho, é possível concluir que o conteúdo estudado na disciplina de programação poderá ser o ferramental para se obter a solução de um determinado problema, utilizando-se estruturas de dados e algoritmos.

A partir deste cenário, o presente artigo apresenta um mapeamento sistemático da literatura brasileira baseado nos principais eventos de informática na educação no Brasil. O objetivo é identificar as metodologias e ferramentas de apoio para o ensino de programação que são utilizados em cursos de introdução à programação na educação básica formal e em universidades. O resultado aponta para a utilização, na sua maior parte, de metodologias e ferramentas baseadas em gamificação, além de um maior número de trabalhos e pesquisas focado na educação básica formal.

\section{Metodologia}

Segundo [19] e [30], o processo de mapeamento sistemático da literatura (MSL) tem como objetivo fazer uma pesquisa em largura na literatura, e não em profundidade. Foram utilizadas as propostas dos autores, que dividem o mapeamento em quatro etapas: (i) Definição das questões de pesquisa; (ii) Execução da pesquisa por estudos primários relevantes; (iii) Extração e síntese dos dados; (iv) Análise dos dados.

Definido o procedimento metodológico que será utilizado na realização desta pesquisa, elaboramos o objetivo geral que é mapear metodologias interdisciplinares para o ensino de programação que são utilizados em cursos de introdução à programação na educação básica e superior. Os objetivos específicos que são (i) Avaliar a evolução histórica das publicações sobre o Ensino de Programação no Brasil, (ii) Verificar quais ferramentas são utilizadas no apoio À metodologia, (iii) Investigar se as ferramentas utilizadas são de livre acesso ou são pagas, (iv) Observar qual o tipo de metodologia utilizada (ex: games, interdisciplinar), (v) Se são utilizados na educação básica ou superior. 
Após definidas as questões de pesquisa, foram mapeadas as fontes. Dado o enfoque brasileiro da pesquisa, foram escolhidos como fontes eventos e periódicos do Brasil voltados para educação e informática. Outra justificativa para a escolha dessas bases é que essa pesquisa servirá de base para um trabalho posterior, onde pretende-se investigar se é possível ensinar programação a partir de um software desenvolvido para editoração de partituras baseado em linha de comando e códigos em cursos de música, levando em consideração o conhecimento de estrutura dos alunos desses cursos. A pesquisa foi realizada de forma manual em cada fonte. As fontes de busca selecionadas foram [33][42][49][50].

Para a pesquisa desenvolvida neste artigo consideramos apenas as publicações dos últimos três anos (2014 - 2016) nas bases WCBIE, RBIE, SBIE, WIE. A busca nas bases de dados teve como string "program*" and "método*" or "method*". Optou-se pela escolha dessa string pois "program" possui o mesmo radical em Português e Inglês e ("método*" or "method*") dando opção de busca em artigos na lingue inglesa e portuguesa.

Os critérios de inclusão que foram utilizados no mapeamento: (i) Conter a string de busca no título ou resumo; (ii) Possuir versão disponível online; (iii) Em caso de artigos que tratavam do mesmo ambiente, apenas o mais recente foi incluído.

A partir dos artigos encontrados, definiu-se os critérios de exclusão para manter assim o foco da pesquisa: (i) Artigos cujo foco principal não eram o ensino de programação; (ii) Artigos sobre utilização fora do ensino formal; (iii) Mapeamentos e revisões sistemáticas sobre o uso de ferramentas específicas e de metodologias específicas. Aplicando a string de busca nas quatro bases de dado, foram localizados 184 artigos. Seguindo os critérios de inclusão e exclusão em cada artigo, restaram 34 artigos $^{1}$.

\section{Discussão dos Resultados}

Seguindo as etapas do mapeamento sistemático, nessa seção será mostrada a síntese dos dados obtidos, juntamente com a discussão e análise dos dados inerentes a cada questão de pesquisa.

\subsection{Evolução histórica das publicações sobre o Ensino de Programação no Brasil.}

Observando a Figura 1, podemos perceber que a evolução histórica de publicações sobre metodologias, e ferramentas sobre o ensino de programação, nos principais eventos e periódicos brasileiros tem apresentado um grande salto. Ao olharmos para o ensino básico, podemos observar que o ensino normalmente é focando na utilização das tecnologias com o intuito de alfabetização digital, ou seja, fornecer o acesso as tecnologias. Para mudar esse cenário, precisamos pensar em um ensino básico com proficiência digital, incluindo o pensamento computacional e a programação. Segundo [48], isso atualmente é uma tendência mundial e existem diversos ambientes para ensinar computação para cada faixa etária.

\footnotetext{
1 Os artigos selecionados foram catalogados com o título, autor e ano e estão disponíveis no link: https://docs.google.com/a/lavid.ufpb.br/spreadsheets/d/1LDKKbe40w7RP0yLcNUH7ZGVhnHtwqjadi9mS16pPio/edit?usp=sharing
} 


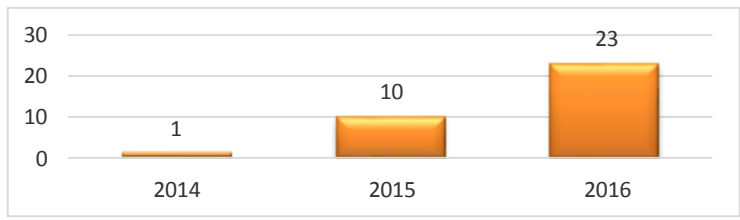

Figura 1. Evolução de publicações nos últimos 3 anos

\subsection{Quais ferramentas são utilizadas no apoio as metodologias?}

Pensar numa metodologia que atraia a atenção do estudante nos momentos iniciais do ensino é de fundamental importância no processo de ensino aprendizagem. Em se tratando de ensino de programação, o uso de ferramentas, ou aplicativos, para a execução de tarefas é indispensável.

Porém, o fato de utilizar alguma ferramenta não garante que os objetivos do processo de ensino aprendizagem sejam alcançados. Diante disso, é fato de que o conhecimento e o domínio do saber são de responsabilidade do professor, entretanto, o uso de aplicações de forma lúdica, e interdisciplinar, poderá ser uma ferramenta pedagógica quando na transposição didática desse saber. Através da técnica, o conhecimento poderá melhor se adequar ao perfil dos nossos alunos na contemporaneidade.

No mapeamento realizado em [2] [4] [5] [6] [13] [14] [16] [17] [24] [25] [29] [31] [32] [35] [36] [38] [40] [41] [43] [45] [46] [48] podemos observar que a evolução no uso de ferramentas com caráter lúdico vem se mostrando cada vez mais forte, focado principalmente em uma programação visual, onde num primeiro momento o código não é o mais importante, e sim o conceito e a lógica de programação.

A Figura 2 mostra as principais ferramentas utilizadas no apoio às metodologias de ensino de programação.

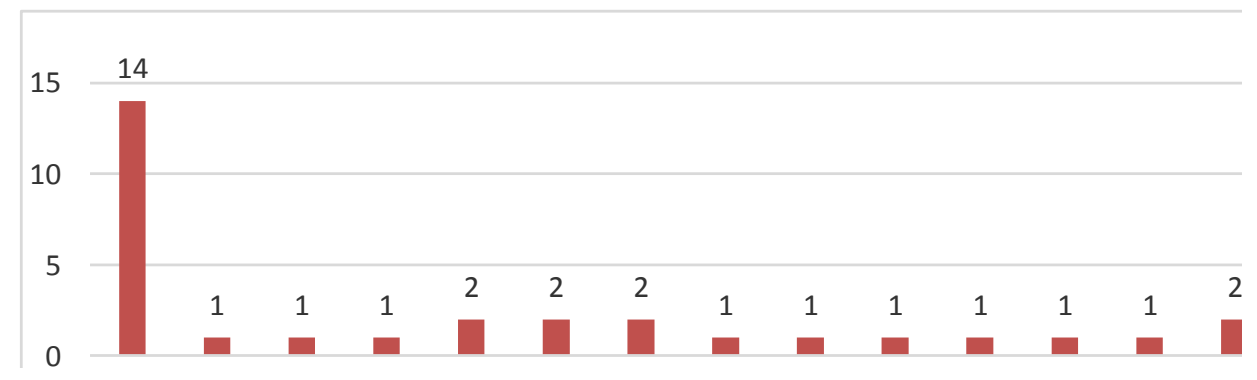

Figura 2. Ferramentas utilizadas como apoio a metodologia de ensino.

Todos os artigos analisados abordam metodologias de ensino de programação, mas alguns não citam a utilização de ferramentas ou aplicativos. Uma das ferramentas mais utilizada é o Scratch [4] [5] [6] [25] [29] [31] [35] [36] [38] [40] [41] [45] [46] [48], que possibilita às crianças elaborem animações, histórias interativas ou jogos. A combinação de gráficos, imagens, fotos, música e som é simples e de fácil aprendizado. Com o Scratch é possível criar personagens que dancem, cantem e interagem uns com os outros. Permite também integrar imagens com efeitos de som e clipes musicais para criar um cartão interativo de aniversário para um amigo ou para criar um mapa interativo. Segundo [32], diversas pesquisas mostram que o uso do Scratch contribui positivamente no ensino de computação em escolas. Criando programas de software com o Scratch, crianças aprendem a pensar criativamente, a trabalhar de forma colaborativa e a pensar de forma sistemática na solução de problemas. 


\subsection{As ferramentas utilizadas são de livre acesso ou são pagas?}

As ferramentas livres no âmbito educacional tornaram-se importantes no combate à exclusão social, uma vez que propiciam a redução de custos na elaboração de laboratórios e de projetos voltados para educação e inclusão digital. Em geral, eles apresentam uma boa qualidade e funcionam em máquinas com configurações de hardware mais antigas, o que ajuda a reduzir os custos.

As publicações encontradas no mapeamento sistemático trazem 17 ferramentas, como o Scratch, App Inventor, Arduino e Code Studio, que são utilizadas para o ensino de programação. Todas são livre ou possuem versões livres. Algumas aplicações têm versões básicas livres e versões completas que necessitam de aquisição para funcionar. Como todos os trabalhos tratam de experimentos com as ferramentas, foram consideradas as versões básicas.

\subsection{Qual o tipo de metodologia utilizada (gamificação, interdisciplinar)?}

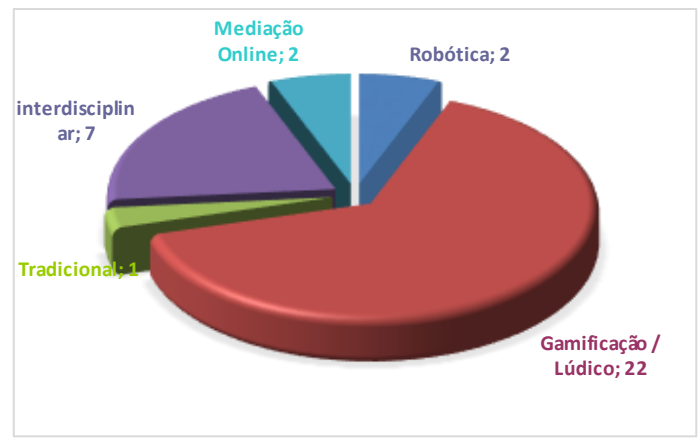

Figura 3. Metodologias que são utilizadas no ensino de programação a partir
do mapeamento.

Como demonstra a Figura3, na grande maioria dos estudos a Gamificação/Lúdico é a metodologia mais utilizada no processo de ensino aprendizagem de programação. O termo gamificação, segundo [18], significa utilizar os mecanismos, a estética e o pensamento dos jogos com o objetivo de encorajar as pessoas, motivar as ações, promover aprendizado e resolver problemas. O termo teve origem na indústria de mídias digitais em 2008, mas foi popularizado somente na segunda metade de 2010 [11].

De acordo com sua definição, a gamificação vem sendo utilizada em sites e aplicativos móveis voltados ao consumidor com a finalidade de convencer as pessoas a utilizarem determinada plataforma. [26] relata que os princípios da gamificação podem ser aplicados em processos de jogos educacionais associando a motivação dos alunos, objetivando melhorar o engajamento e interesse do aprendiz. A gamificação é sustentada a partir dos princípios do design de jogos. Esses princípios definem o que são jogos e como eles funcionam, bem como os elementos do design que os compõem. Os jogos educacionais destacam-se como ferramentas que facilitam no ensino e aprendizagem dos usuários, no entanto esta deve estar interligada à outros recursos de aprendizagem [26].

Em segundo lugar, a metodologia utilizada é a interdisciplinar, com 7 trabalhos na área, tendo como princípio a ligação com outras áreas do conhecimento, como matemática, física e música. A forma tradicional, mediação online e robótica, aparecem com dois trabalhos em cada área. 
VI Congresso Brasileiro de Informática na Educação (CBIE 2017)

Anais do XXIII Workshop de Informática na Escola (WIE 2017)

\subsection{São utilizados na educação básica ou superior?}

A introdução de conceitos de Computação, enquanto ciência, na educação básica é de fundamental importância e relevância [10]. No entanto, seu ensino, no Brasil, por muitos anos tem tido enfoque nos âmbitos da graduação e pós-graduação. Atualmente vemos uma grande mudança nesse sentido, pois com o desenvolvimento tecnológico, a facilidade de acesso e o incentivo do poder público têm mudado esse paradigma. O ACM Model Curriculum for K-12 Computer Science - [10] defende que é necessário o desenvolvimento de habilidades computacionais na Educação Básica, no sentido de a Ciência da Computação ser importante intelectualmente, promover múltiplos caminhos profissionais futuros, desenvolver a capacidade de resolver problemas, apoiar e relacionar-se com outras ciências e motivar os estudantes.

Neste mapeamento vemos que grande parte dos trabalhos de pesquisa se desenvolvem na educação básica [2] [4] [5] [8] [13] [14] [17] [22] [23] [24] [25] [28] [29] [34] [40] [41] [45] [46] [47] [48], visando a introdução dos elementos computacionais de forma lúdica, com os mais diversos objetivos, como ajudar na aprendizagem de conteúdos de matemática.

A Figura 4 mostra em quais níveis as pesquisas são realizadas.

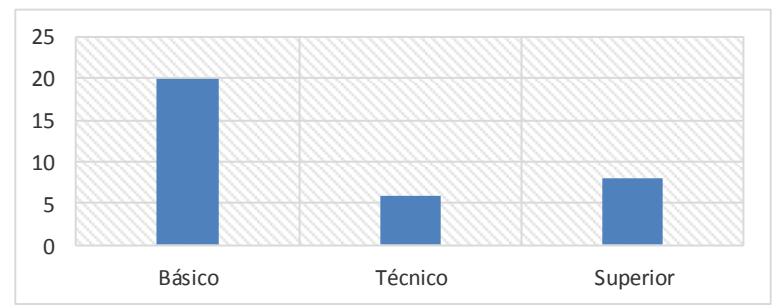

Figura 4. Níveis de ensino onde as pesquisas são realizadas

\section{Considerações Finais}

Esse artigo apresentou um mapeamento sistemático que permite analisar as pesquisas brasileiras com relação às metodologias utilizadas no ensino da programação e quais ferramentas dão apoio a essa metodologia. $\mathrm{O}$ mapeamento se baseou em publicações dos principais veículos nacionais da área de Informática na Educação. Com a análise dos dados extraídos do mapeamento foi possível observar a evolução histórica das publicações, responder quais são as ferramentas utilizadas, se são de livre acesso ou pagas, qual o tipo de metodologia utilizada, e se são utilizados na educação básica ou superior. Observou-se o aumento no número de publicações relacionadas a ambientes de ensino de programação no ano de 2016. É importante destacar que todos os artigos selecionados foram escritos em Língua Portuguesa.

Outro ponto importante descoberto durante a análise dos resultados foi a preferências dos professores em utilizar o Scratch como ferramenta de apoio, pois tem um caráter lúdico para o ensino de programação. Tal fato está diretamente ligado à tendência da Gamificação das ferramentas, situação que prende mais a atenção do aluno.

Os espaços onde os estudos são desenvolvidos também chamam a atenção, pois durante muitos anos o ensino de programação estava restrito às universidades. Com o desenvolvimento tecnológico recente, e os incentivos governamentais é possível perceber que atualmente o ensino de programação está presente também na educação básica. 
VI Congresso Brasileiro de Informática na Educação (CBIE 2017)

Anais do XXIII Workshop de Informática na Escola (WIE 2017)

\section{Referências}

[1] Albuquerque, D.; Bremgartner, V.; Lima, H.; Salgado, N.; Uma Experiência do Uso Do Hardware Livre Arduino no Ensino De Programação De Computadores - In V Congresso Brasileiro de Informática na Educação (CBIE 2016);

[2] Amaral, L. R. do; Silva, G. B. e; Pantaleão, E. Plataforma Robocode como Ferramenta Lúdica de Ensino de Programação de Computadores - Extensão Universitária em Escolas Públicas de Minas Gerais - In Anais do XXVI Simpósio Brasileiro de Informática na Educação (SBIE 2015);

[3] Aureliano, V. C. O. and Tedesco, P. C. A. R. Ensino e Aprendizagem de Programação para Iniciantes: uma Revisão Sistemática da Literatura focada no SBIE e WIE. Simp. Brasileiro de Inf. Na Educação, 2012;

[4] Batista, E. J. S.; Castro Jr., A. A.; Bogarim, C. A. C.; Larrea, A. A. Utilizando o Scratch como ferramenta de apoio para desenvolver o raciocínio lógico das crianças do ensino básico de uma forma multidisciplinar - In Anais do XXI Workshop de Informática na Escola (WIE 2015);

[5] Batista, E. J. S.; Castro Jr., A. A.; Cantero, S. V.; Bogarim, S. A. C.; Larrea, A. A. Uso do Scratch no ensino de programação em Ponta Porã: das séries inicias ao ensino superior - In Anais do XXII Workshop de Informática na Escola (WIE 2016);

[6] Belchior, H.; Bonifácio, B.; Ferreira, R. Avaliando o Uso da Ferramenta Scratch para Ensino de Programação através de Análise Quantitativa e Qualitativa -In Anais do XXVI Simpósio Brasileiro de Informática na Educação (SBIE 2015);

[7] Cambruzzi, E.; Souza, R. M. de. Robótica Educativa na aprendizagem de Lógica de Programação: Aplicação e análise - In Anais do XXI Workshop de Informática na Escola (WIE 2015);

[8] Cardoso, R.; Antonello, S. L. Interdisciplinaridade, programação visual e robótica educacional: relato de experiência sobre o ensino inicial de programação - In Anais dos Workshops do IV Congresso Brasileiro de Informática na Educação (CBIE 2015);

[9] Carvalho, L. S. G.; Oliveira, D. B. F.; Gadelha, B. F. Juiz online como ferramenta de apoio a uma metodologia de ensino híbrido em programação - In Anais do XXVII Simpósio Brasileiro de Informática na Educação (SBIE 2016);

[10] CSTA - Computer Science Teacher Association. (2011) "CSTA K-12 Computer Science Standards". CSTA Standards Task Force. ACM - Association for Computing Machinery;

[11] Deterding, S.; Dixon, D.; Khaled, R.; Nacke, L. E. Gamification: Toward a Definition. Conference on Human Factors in Computing Systems. Anais.p.12-15, 2011. Vancouver: ACM Press.

[12] EDUCAÇÃO, M. DA. Diretrizes Curriculares Nacionais para os cursos de graduação na área da Computação. v. 2016, n. 1977, p. 1-9, 2012;

[13] Galdino, C. B. T.; Neto, S. R. S.; Costa, E. B. KidCoder: Uma Proposta de Ensino de Programação de forma Lúdica - In Anais do XXVI Simpósio Brasileiro de Informática na Educação (SBIE 2015);

[14] Gomes, R. C. S.; Melo, J. C. B. de; Tedesco, P. C. A. R. Jogos Digitais no Ensino de Conceitos de Programação para Crianças - In Anais do XXVII Simpósio Brasileiro de Informática na Educação (SBIE 2016); 
VI Congresso Brasileiro de Informática na Educação (CBIE 2017)

Anais do XXIII Workshop de Informática na Escola (WIE 2017)

[15] Greening, T. Emerging Constructivist Forces in Computer Science Education: Shaping a New Future? Computer Science Education in the 21st Century, p. 47-80, 2000;

[16] Izeki, C. A.; Nagai, W. A.; Dias, R. M. C. Experiência no Uso de Ferramentas Online Gamificadas na Introdução à Programação de Computadores - In Anais do XXII Workshop de Informática na Escola (WIE 2016)

[17] Jesus, A. M. de; Silva, G. M. da DOJO GO: Uma Fo;rma Divertida e Colaborativa de se Aprender através de Dojo de Programação de Jogos - In Anais do XXII Workshop de Informática na Escola (WIE 2016);

[18] Kapp, K. M. The Gamification of Learning and Instruction. Pfeiffer Publishing, 2012.;

[19] Kitchenham, B. and Charters, S. (2007). Guidelines for performing systematic literature reviews in software engineering.;

[20] Lopes, B. G.; Duarte, W. S.; Nogueira, R.C.; Lopes,R. F. F. ; Ferreira, D.J.Método de Ensino de Programção Mediada por Simulação: Um Estudo de Caso no Curso Técnico Integrado em Informática - In Anais do XXVII Simpósio Brasileiro de Informática na Educação (SBIE 2016);

[21] Lopes, C. C.; Duarte, M. S. S. G.; Sousa, E. A. D.; Souza, E. P. de, Pereira, I. B. O Ensino de Algoritmos e Lógica de Programação como uma Ferramenta Pedagógica para Auxiliar a Aprendizagem de Matemática: Um Relato de Experiência - In Anais dos Workshops do V Congresso Brasileiro de Informática na Educação (CBIE 2016);

[22] Marinho, C. S. S.; Moreira, L. O.; Coutinho, E. F.; Paillard, G. A. L.; Neto, E. T. L. Experiências no Uso da Metodologia Coding Dojo nas Disciplinas Básicas de Programação de Computadores em um Curso Interdisciplinar do Ensino Superior - In Anais dos Workshops do V Congresso Brasileiro de Informática na Educação (CBIE 2016);

[23] Martins, L. A. S.; Brelaz, A. S.; Nascimento, G. R.; Alfaia, R. M.; Martins, T.S. Ensinando Lógica de Programação aplicada à Robótica para alunos do Ensino Fundamental - In Anais do XXVII Simpósio Brasileiro de Informática na Educação (SBIE 2016);

[24] Martins, R. S.; Reis, R. J. A. dos; Marques, A. B. Inserção da programação no ensino fundamental: Uma análise do jogo Labirinto Clássico da Code.org através de um modelo de avaliação de jogos educacionais - In Anais do XXII Workshop de Informática na Escola (WIE 2016);

[25] Mattos, F. de; Ferreira, V.; Anacleto, J. O Ensino de Programação de SCRATCH E Seu Impacto na Opção Profissional para as Meninas - In Anais do XXVII Simpósio Brasileiro de Informática na Educação (SBIE 2016);

[26] Muntean, C. I. Raising engagement in e-learning through gamification. The 6th International Conference on Virtual Learning ICVL 2011. Anais. p.323-329, 2002.

[27] Nagano, L. H.; Direne, H. I. Ensino de lógica de programação baseado na indução-dedução através de exemplos - In Anais do XXVII Simpósio Brasileiro de Informática na Educação (SBIE 2016);

[28] Oliveira, C. E. T. de; Nogueira, E. C.; Motta, C. L. R. da; Meireles, L. B. Relação estudante-professor: Educação Baseada na Construção de Jogos - In Anais do XXVI Simpósio Brasileiro de Informática na Educação (SBIE 2015); 
VI Congresso Brasileiro de Informática na Educação (CBIE 2017)

Anais do XXIII Workshop de Informática na Escola (WIE 2017)

[29] Oliveira, M. V. de; Rodrigues, L. C.; Queiroga, A. P. G. de Material didático lúdico: uso da ferramenta Scratch para auxílio no aprendizado de lógica da programação - In Anais do XXII Workshop de Informática na Escola (WIE 2016);

[30] Petersen, K., Feldt, R., Mujtaba, S., and Mattsson, M. (2008). Systematic mapping studies in software engineering. In Proceedings of the 12th International Conference on Evaluation and Assessment in Software Engineering, EASE'08, pages 68-77, Swinton, UK, UK. British Computer Society.;

[31] Rafalski, J. P.; Santos, O. L. dos Uma experiência com a Linguagem Scratch no Ensino de Programação com Alunos do Curso de Engenharia Elétrica - In Anais do XXII Workshop de Informática na Escola (WIE 2016);

[32] Resnick M. et al. Scratch: programming for all. Communications of the ACM, 52(11), 2009, pp. 60-67.;

[33] Revista Brasileira de Informática na Educação - RBIE);

[34] Ribeiro, J. P.; Manso, M. A.; Borges, M. A. F. Dinâmicas com App Inventor no Apoio ao Aprendizado e no Ensino de Programação - In Anais do XXII Workshop de Informática na Escola (WIE 2016);

[35] Rodrigues, L.C.; Queiroga, A. P. G. de; Oliveira, M. V. de; More, A. T. Relato de experiência: curso de introdução à programação para crianças do ensino fundamental no IFSP Votuporanga - In Anais do XXII Workshop de Informática na Escola (WIE 2016);

[36] Salazar, R.; Odakura, V.; Barvinski, C. Scratch no ensino superior: motivação - In Anais do XXVI Simpósio Brasileiro de Informática na Educação (SBIE 2015);

[37] Salgado, L. A. Z. Desprogramamdo Programado. Anais do VII Simpósio Nacional da ABCiber, n. X, p. 1-11, 2013.;

[38] Santiago, A. D. V.; Kronbauer, A. H. Um Modelo Lúdico para o Ensino de Conceitos de Programação de Computadores- In Anais do XXVII Simpósio Brasileiro de Informática na Educação (SBIE 2016);

[39] Santos, A. C. T. dos; Monteiro, J. A.; Machado, K. C. T.; Lins, P. R. B.; Ramos, T. A. R.; Batista, L. V. Ensino de programação para Olimpíada Brasileira de Informática - In Anais do XXI Workshop de Informática na Escola (WIE 2015);

[40] Silva, D. O. da; Garcia, V. R.; Oliveira, I. G. de; Trindade, D. F. G.; Sgarbi, E. M.; Nascimento, L. F. L. Despertando Jovens Talentos com o Conhecimento da Computação In Anais do XXII Workshop de Informática na Escola (WIE 2016);

[41] Silva, T. R. da; Aranha, E. H. S.; Santos, F. G.; Tavares, K. F. Um Relato de Experiência da Aplicação de Videoaulas de Programação de Jogos Digitais para Alunos da Educação Básica - In Anais do XXII Workshop de Informática na Escola (WIE 2016);

[42] Simpósio Brasileiro de Informática na Educação - SBIE;

[43] Souza, M. S. C. de; Costa, F. A. M.; Silva, V. L.; Terra, D. C. Lord of Code: uma ferramenta de apoio ao ensino de programação In Anais do XXVII Simpósio Brasileiro de Informática na Educação (SBIE 2016);

[44] Souza, N. S. de. Uma Abordagem Dialética Para a Pesquisa Interdisciplinar em Interação Humano-Computador. p. 150, 2016; 
VI Congresso Brasileiro de Informática na Educação (CBIE 2017)

Anais do XXIII Workshop de Informática na Escola (WIE 2017)

[45] Souza, P. S. S. de; Mombach, J. G. Ensino de Programação para Crianças através de Práticas Colaborativas nas Escolas - In Anais do XXII Workshop de Informática na Escola (WIE 2016);

[46] Teixeira, A. C.; Oro, N. T.; Batistela, F.; Martins, J. A. R.; Pazinato, A. M. Programação de computadores para alunos do ensino fundamental: A Escola de Hackers - In Anais do XXI Workshop de Informática na Escola (WIE 2015);

[47] Vera, W. F. M.; Miranda, A. M.; Costa, W. N.; Matos, E. S. Dó, Ré, Mergesort: um relato de experiência interdisciplinar de ensino de computação com matemática e música - In Anais dos Workshops do V Congresso Brasileiro de Informática na Educação (CBIE 2016);

[48] Wangenheim, C. G. von; Nunes, V. N.; Santos, G. D. dos. Ensino de Computação com SCRATCH no Ensino Fundamental - Um Estudo de Caso. Revista Brasileira de Informática na Educação - RBIE V.22 N.3 - 2014;

[49] Workshop de Informática na Escola - WIE (http://brie.org/pub/index.php/wie/index);

[50] Workshops do Congresso Brasileiro de Informática na Educação - WCBIE;

[51] Zaina, L. A. M. Projeto Multidisciplinar: uma Experiência Prática no Ensino de Programação em um Curso de Engenharia da Computação. Xxxiii - Congresso Brasileiro de Ensino de Engenharia, 2005. 